Manch ein Sportler kann vom Training nicht genug bekommen. Den Spaß am Sport etwas dämpfen dürfte allerdings die Erkenntnis, dass extremes Training das Risiko für Vorhofflimmern deutlich erhöht.

Ausdauersportler wie Skilangläufer sind deutlich anfälliger für Vorhofflimmern als die Normalbevölkerung. Herausgefunden haben das norwegische Forscher um Dr. Lostein Grimsmo von der Feiring Herz Klinik in Norwegen. Sie begleiteten über 30 Jahre Sportler, die regelmäßig am Birkebeiner Querfeldein-Ski-Marathon teilnahmen. Ein Wettkampf, bei dem sich die Langlauf-Elite mit einem wenigstens $3,5 \mathrm{~kg}$ schweren Rucksack über eine $54 \mathrm{~km}$ lange Schneepiste hetzt. Die für die Studie ausgewählten Teilnehmer stellten sich von 1976 an in größeren Abständen für eine kardiologische und pulmologische Funktionsprüfung zur Verfügung.
Über die Jahre dokumentierten die Forscher unter den Probanden mit 12,8\% eine so hohe Rate an Vorhofflimmern bereits im mittleren Alter von 58 Jahren, wie sie zuvor noch nie bei unter 75-Jährigen nachgewiesen wurde. Eigentlich liegt diese bei nur 0,5\%, bei über 75Jährigen liegt sie bei 15\%. Als Prädiktoren für die erhöhte Prävalenz fanden die Forscher zwei bei Hochtrainierten typische Eigenschaften: den niedrigen Ruhepuls und den vergrößerten linken Vorhof. Den Spaß im Schnee sollten sich Sportler also nicht zu sehr zu Herzen nehmen.

(ch)

Grimsmo J et al, Eur J Cardiovasc Prev Reha 2010, 17:100

\section{Weinen mit der Speicheldrüse}

Was hat eine Unterkieferspeicheldrüse in der Schläfe zu suchen? Ganz einfach: Sie soll durch ihr Sekret die Augen befeuchten, wenn das Auge selbst dies nicht ausreichend schafft. Seit Mitte der 90er-Jahre wird diese Transplantation bei Patienten mit trockenem Auge mit der eigenen Speicheldrüse schon vorgenommen. Jetzt hat ein Lübecker Chirurgen-Team einem 43-jährigen Patienten erstmals die Drüse eines Fremdspenders nämlich seines Bruders - verpflanzt. Pressemitteilung Campus Lübeck, 5.2.2010

\section{Fischöl gegen Schizophrenie}

Einer drohenden Psychose können möglicherweise Fischöl-Kapseln entgegenwirken. Die darin enthaltenen ungesättigten Fettsäuren beeinflussen die Freisetzung von Neurotransmittern, die an der Entstehung von Schizophrenie beteiligt sind. So erkrankten in einer Studie nur 4,5\% der psychosegefährdeten Teilnehmer mit „FischölPrävention“" an einer manifesten Psychose; dagegen kam es bei 27,5\% ohne vorbeugende Fettsäuren zum Ausbruch der Schizophrenie.

Amminger G, Arch Gen Psychiatry 2010, 67:146
9 CME-Punkte kostenlos in jeder Ausgabe

\author{
CME hilft Ihnen, Ihr \\ Punktekonto zu füllen!
}

Mit unseren drei zertifizierten

Fortbildungen können Sie pro Heft auf CME.springer.de/CME kostenlos 9 Punkte sammeln. Und so geht's:

\section{Kostenlos teilnehmen in vier Schritten}

\section{Auswählen}

Wählen Sie online auf CME.springer. de/CME den Link zum aktuellen Heft aus. Entscheiden Sie nun, welche Fortbildungseinheiten Sie zum Erwerb von CME-Punkten nutzen wollen. Die Teilnahme an den drei Einheiten der aktuellen Ausgabe ist kostenlos.

\section{Registrieren/Anmelden}

Sind Sie bereits bei CME.springer.de registriert? Dann genügt zur Anmeldung die Angabe Ihrer persönlichen Zugangsdaten. Falls Sie zum ersten Mal teilnehmen, bitten wir Sie, sich einmalig zu registrieren. Wir senden Ihnen dann per E-Mail Ihre persönlichen Zugangsdaten zu. Bitte benutzen Sie diese für alle weiteren Teilnahmen zur Anmeldung (Login).

\section{Teilnehmen}

Sie können die elektronische Version der Fortbildung online lesen, ausdrucken oder direkt die 10 CME-Fragen beantworten. Die kostenlose Teilnahme ist 3 Monate nach Erscheinen der Zeitschrift möglich.

\section{CME-Punkte sammeln}

Nach erfolgreicher Beantwortung von 7 der 10 CME-Fragen senden wir Ihnen Ihre Teilnahmebestätigung samt Punkten umgehend per E-Mail zu. Diese können Sie bei Ihrer zuständigen Landesärztekammer einreichen. 